\title{
Major Outbreak of Dermatophyte Infections Leading Into Imitation of Different Skin Diseases: Trichophyton Mentagrophytes is the Main Criminal Fungus
}

\author{
(1) Khalifa E. Sharquie', (1) Raed I. Jabbar ${ }^{2}$ \\ ${ }^{1}$ University of Baghdad College of Medicine, Medical City Teaching Hospital, Department of Dermatology, Baghdad, Iraq \\ 2Fallujah Teaching Hospital, Al-Anbar Health Directorate, Department of Dermatology, Anbar, Iraq
}

\section{ABSTRACT}

Background: It is well known that fungal skin infections lead into different clinical presentations but when some countries face epidemic or outbreak of infection, this will lead into more confusions in clinical presentation, so more new odd clinical faces of the disease.

Materials and Methods: This is a case series study where 500 cases with misdiagnosis or suspicion of different skin diseases but with suspicion of fungal skin infections that presenting with unusual clinical pictures were collected during the period from 2016-2021 years. Skin samples from lesions of the cases including scales and hair were collected and examined by direct microscopic examination. In addition, skin scraping and hair were taken from 92 patients (as a sample for culture and/or polymerase chain reaction) with suspicion and misdiagnosis of dermatophyte infections during the period from April to December 2019, for mycological diagnostics and sequencing.

Results: This study included 500 patients, 298 (59.6\%) male and 202 (40.4\%) females with varying types of dermatophyte infections. All patients had atypical clinical presentations with prior misdiagnosis of different non-fungal skin diseases. Dermatophyte infections were mimicking the following diseases: Psoriasis-like lesion (50.2\%) was the most common clinical presentation while dermatitis-like seen in 107 (21.4\%) patients, photosensitivity-like in 24 (4.8\%) cases,keratoderma in 17 (3.4\%), seborrheic dermatitis-like in 14 (2.8\%), napkin dermatitislike in 12 (2.4\%), folliculitis-like in $12(2.4 \%)$, rosacea-like in $12(2.4 \%)$, moth eaten alopecia in 11 (2.2\%), lupus erythematosus-like in 10 (2\%), discoid lupus erythematosus-like in 8 (1.6\%), granuloma annularae-like in 4 (0.8\%), pityriasis alba-like in $3(0.6 \%)$ and others.

Conclusion: Iraq is running major outbreak of dermatophyte infections that have bizzare clinical pictures and imitating many skin diseases commonly psoriasis, dermatitis, photosensivity and keratoderma. Trichophyton mentagrophytes represented the most frequent dermatophyte that has been isolated.

Keywords: Atypical presentation, Dermatophytosis, Zoophilic, Anthropophilic

\section{Introduction}

Dermatophytes are the most common agents of superficial fungal infections all over the world and widespread in the developing countries [1].
These fungi possess affinity for keratinized tissue, and can cause superficial infections of the skin, nails and hair. Trichophyton (T), epidermophyton (E), and microsporum (M) are the three main genera of dermatophytes that cause dermatophytosis [2]. 
Currently, fungal infections of the skin, hairs and nails have become a common ecumenical dilemma. Havlickova et al. [3], 2008 reported that $20-25 \%$ of the world's population has skin mycoses and appeared to be a frequent form of infection.

There is no more distinguishing between zoophilic and anthropophilic fungi regarding infection to the human being. So both types can infect humans and when zoophilic infect human then human can infect another human [2].

In the last year's zoophilic fungi replacing anthropophilic fungi as the etiological pattern of dermatophytosis $[4,5,6,7,8,9,10]$. The incidence and distribution of this infection have been studied in different geographical areas of the world including Iraq [11,12], Kuwait [13], Jordan [14], Saudi Arabia [15], Greece [16] and Europe [17].

Dogra and Narang [18] in 2017 reported cases with unusual presentation of dermatophytosis in Indian peoples which are termed as atypical tinea. Atypical tinea can be seen even in immunocompetent patients who are not using any topical and systemic medications such as corticosteroids.

Superficial fungal infection of the skin may sometimes imitate other dermatoses and multiple atypical clinical varieties have been recorded which include eczematous dermatitis-like, psoriasis-like, impetigo-like, seborrheic dermatitis-like, erythema multiformelike, keratoderma-like, pyoderma gangrenosum-like lupus erythematosus-like, lichen planus like, dermatitis herpetiformislike, rosacea-like, herpes-like, and polymorphous light eruption-like $[18,19,20]$.

There is an authentic epidemic of varieties of chronic recalcitrant cutaneous fungal infection due to T. mentagrophytes (TM) type VIII in India [21] and a wide variation in clinical pictures is seen. Tinea corporis, tinea faciei and tinea cruris are the most common presentations. Lesions usually show mild to severe degree of inflammation, and huge lesions with a tendency to coalesce and spread are common [22].

Numerous reports and literature recorded that TM type VIII is becoming increasingly prevalent in many countries. TM type VIII has been isolated from skin scrapings of patients in Iraq, Germany, Switzerland, Finland, Cambodia, Estonia and Iran [23,24,25,26,27].

As Iraq is running an outbreak of dermatophyte infections leading into epidemic state, new strange clinical presentations are expected. So the aim of present work is to record all abnormal and odd cases of fungal skin infections that had imitated other skin diseases.

\section{Materials and Methods}

This is a case series study carried out during the period from 2016-2021. The present study included any patients with positive potassium hydroxide $(\mathrm{KOH})$ examination of the suspected cutaneous fungal infection, those with suspected fungal infection but with negative $\mathrm{KOH}$ examination were excluded from the study. Patients from northern to southern Iraqi governorate were involved in the present study but the majority of the patients were from the capital Baghdad. Oral consent was obtained from each patient after explanation of the nature of the study. Close-up photographs were taken at the same place with constant distance and illumination.

Demographic data and detailed disease history were gathered from all patients. Visual inspection with appropriate light source and proper dermatological examination including woods light examination were carried out yielding different clinical types of superficial fungal infection. Family members living in the same house were also examined for any suspected skin lesions.

All cases presented with unusual clinical pictures that could be easily missed with other skin diseases. The following diagnostic parameters were applied to have higher index of suspicion in favor of fungal infections: unusual sites, scaly lesions with hair loss, bizarre forms with arches and angulations, geographical in shapes, fire in field pictures, target wavy lesions, asymmetry of lesions, psoriasis of scalps with patchy alopecia, unusual ages. Also increasing number of cases in the same family or close relatives, higher number among the same society.

The diagnosis was based on clinical features confirmed with direct microscopic examination (10-20\% $\mathrm{KOH})$. Skin scrapings from the edge of the lesion, infected hair, nail clippings and debris from under the edges of the infected nails were gathered. Direct microscopic examination using $\mathrm{KOH}$ of the skin scrapings, hair and/ or nail clippings was done in all cases after immersion in $10 \% \mathrm{KOH}$ for skin scales and hair examination and $20 \% \mathrm{KOH}$ for nail specimens examination.

In addition, skin scraping and hair were taken from 92 patients, [as a sample for culture and or polymerase chain reaction (PCR)], with suspicion and misdiagnosis of dermatophyte infections during the period from April to December 2019. Skin scraping and hair were collected in the Center of Dermatology, Medical City Teaching Hospital, Baghdad, Iraq and sent to Laboratory of Medical Microbiology, Mölbis, Germany for mycological diagnostics and sequencing [24].

The patients were divided into several groups according to the clinical presentation: psoriasis-like, dermatitis-like, rosacea-like, photosensitivity-like, seborrhiec dermatitis-like, keratoderma of palms and/ or soles and others.

\section{Statistical Analysis}

Statistical Package for Social Sciences version 23 was used for data input and analysis. Data were statistically described in terms of 
mean, frequencies (number of cases), standard deviation, male to female ratio and percentage (\%).

\section{Results}

This study included 500 patients with dermatophyte infections, 298 (59.6\%) male and 202 (40.4\%) females with varying types of dermatophyte infections, their ages ranged from 4 months-70 years with median of 30 years. The duration of the disease ranged from 2-36 months with a mean of 4 months. The number of familial house contacts were ranged from 1-30 with a median of 5 cases.

All patients had atypical clinical presentation (Figures 1-8) with prior misdiagnosis or suspicion of different non-fungal skin diseases receiving various topical and systemic therapies while $\mathrm{KOH}$ examination was positive for all included patients. Both by PCR and/or culture for the 92 patients, dermatophyte was detected in 63 (68\%) out of 92 samples. PCR positive were 57 (90\%) of 63 samples, culture positive was 43 (81\%). The dermatophyte species isolated were belonged to the 3 genera T, E and M. Eight dermatophytes

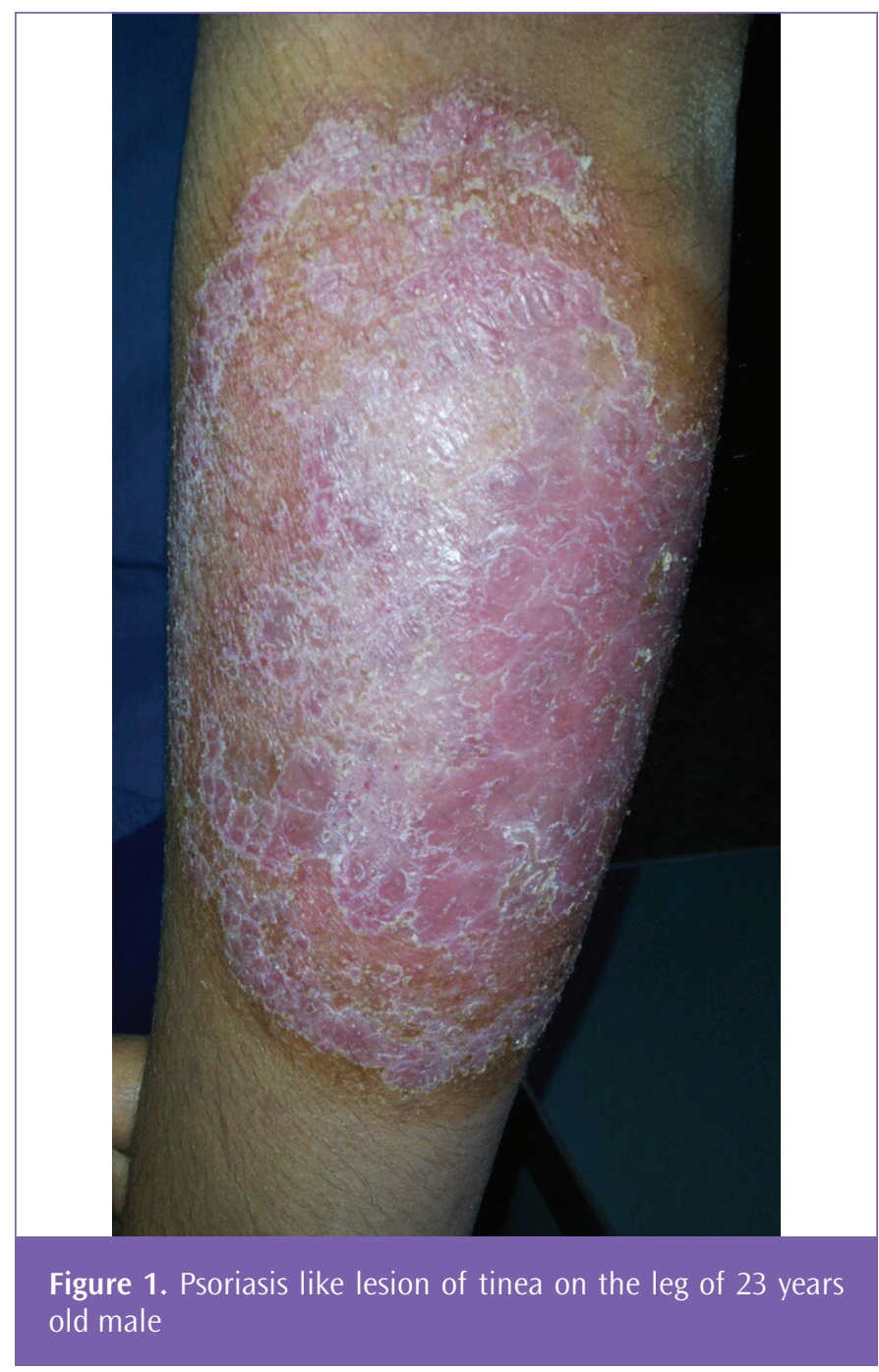

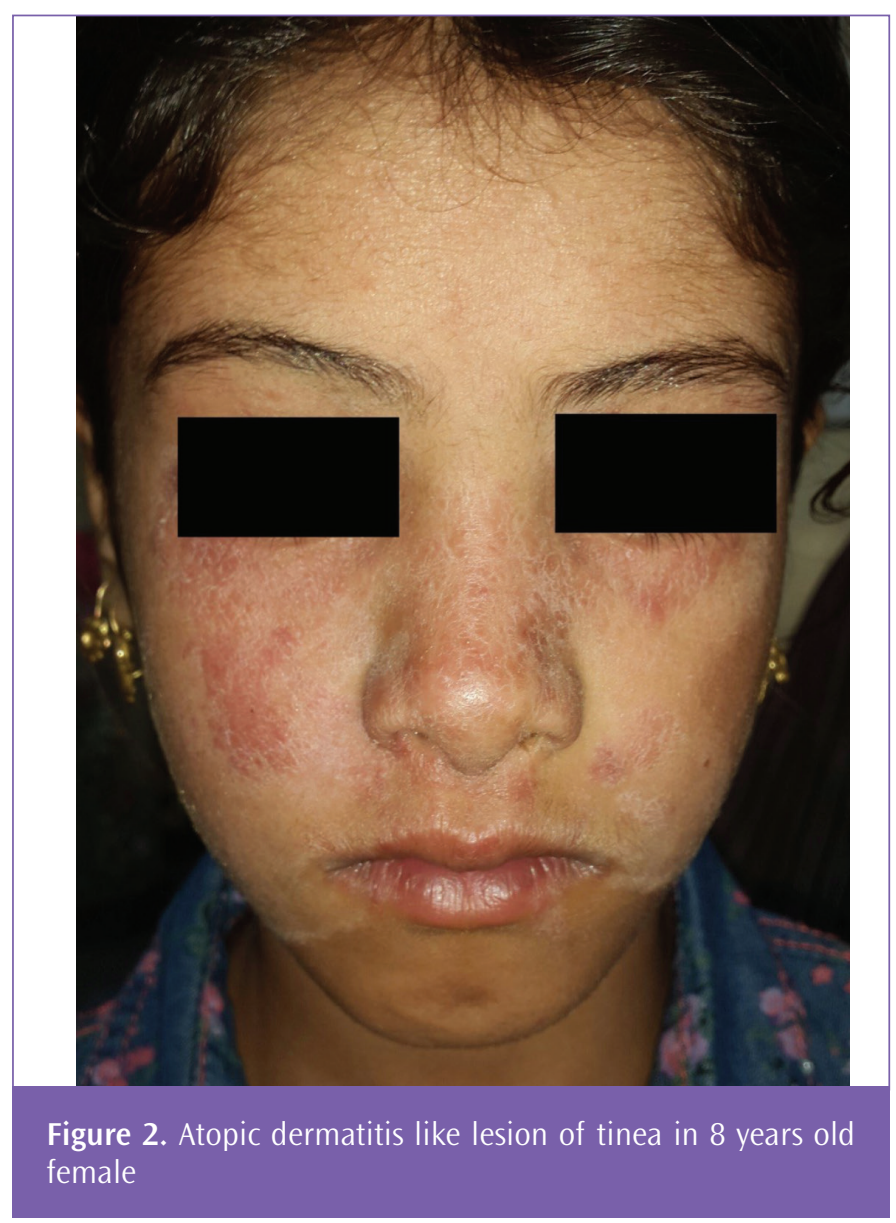

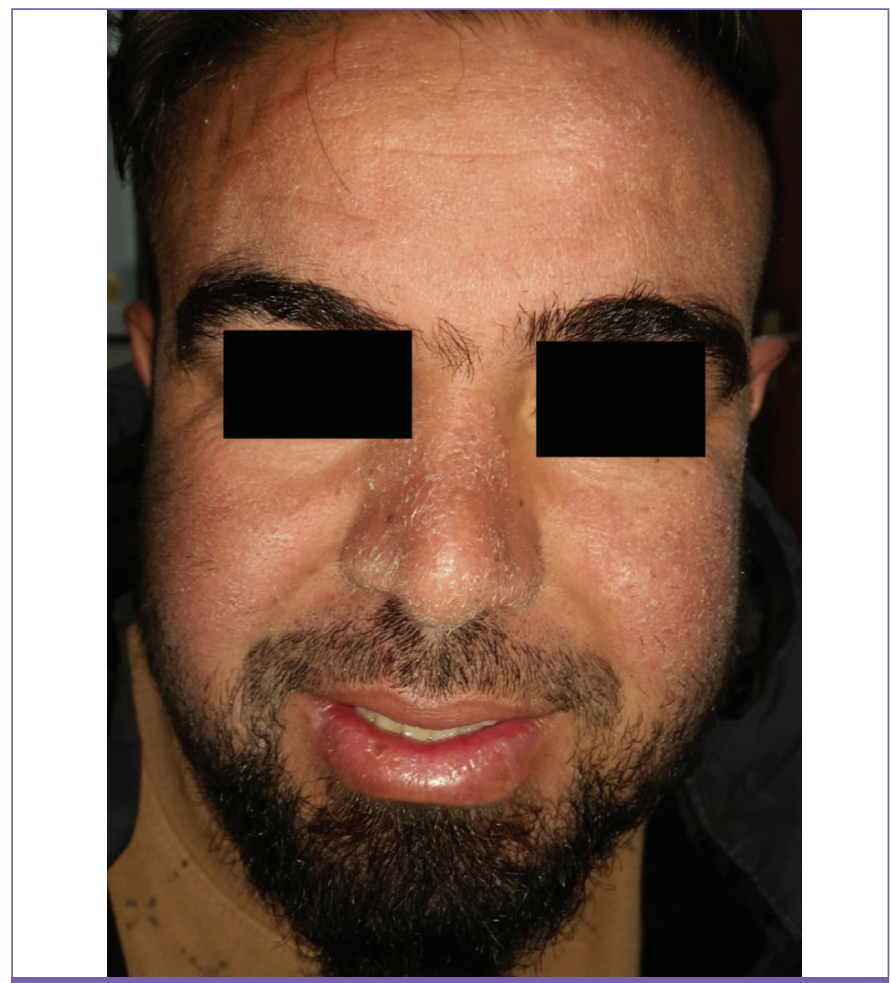

Figure 3. Seborrhiec dermatitis like lesion of tinea on the face of 21 years old male 


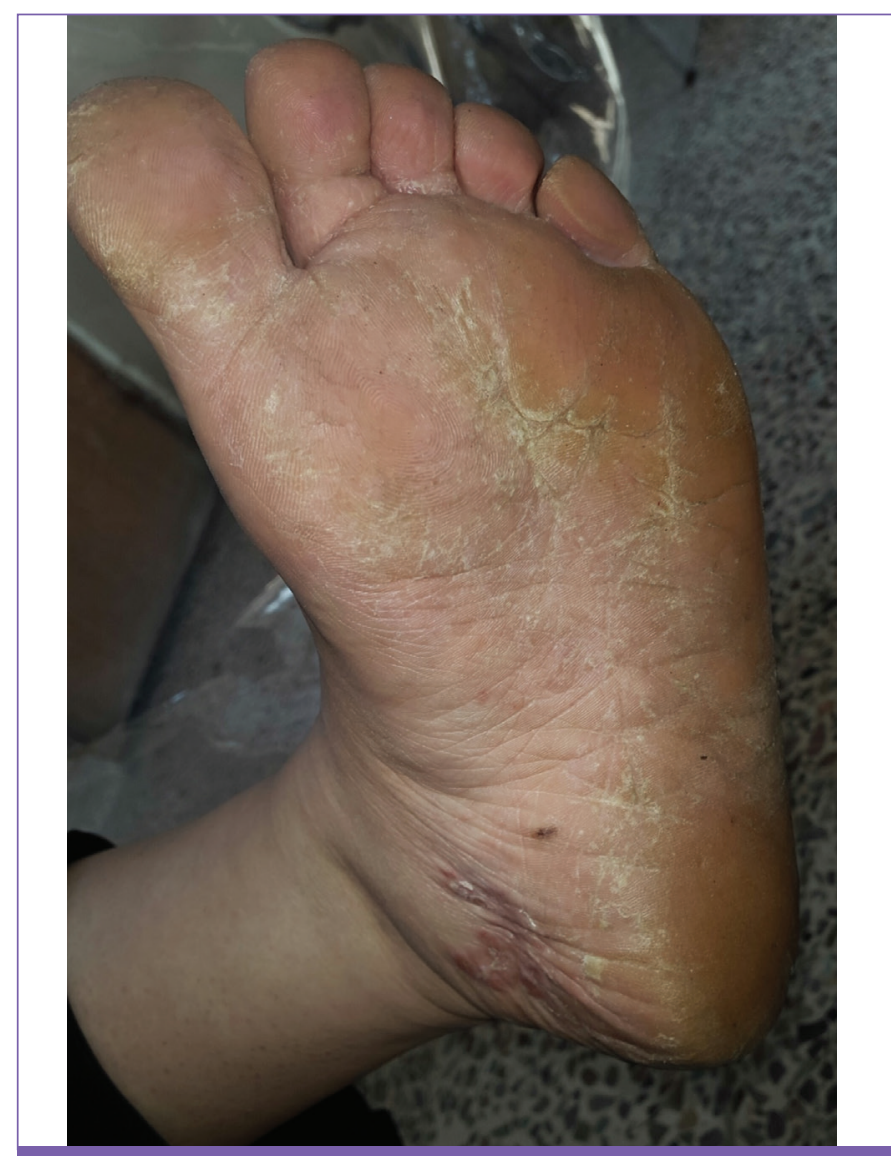

Figure 4. Left foot keratoderma lesion of tinea in 40 years old female

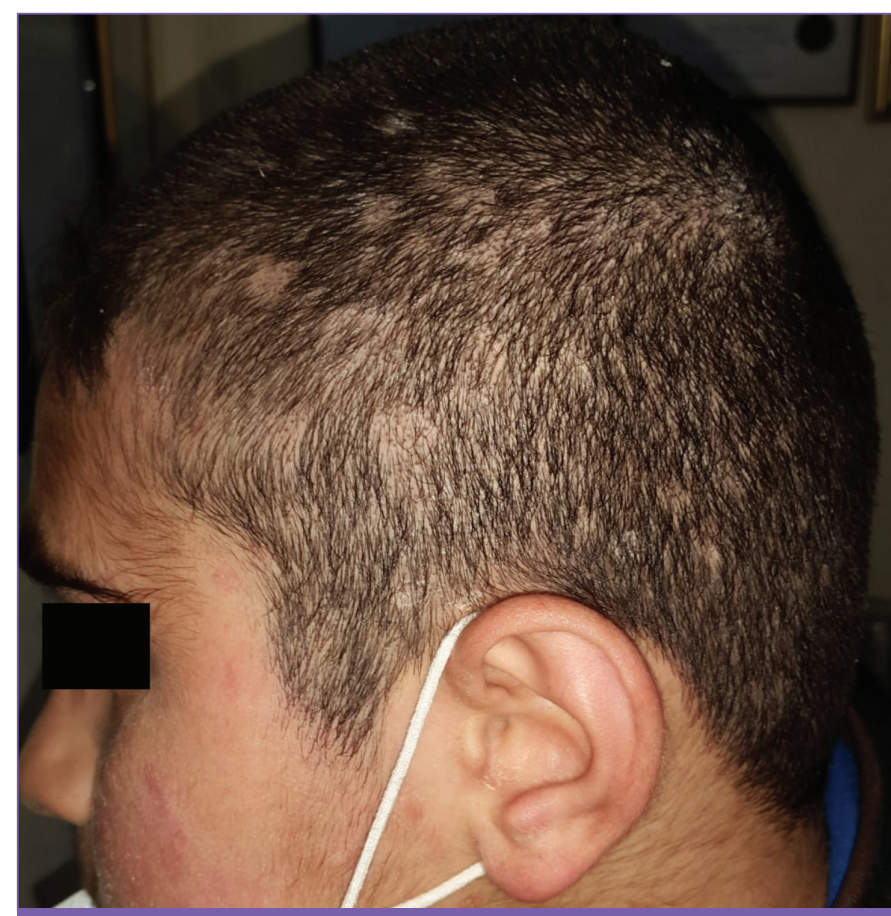

Figure 5. Moth eaten alopecia of tinea capitis in 11 years old male

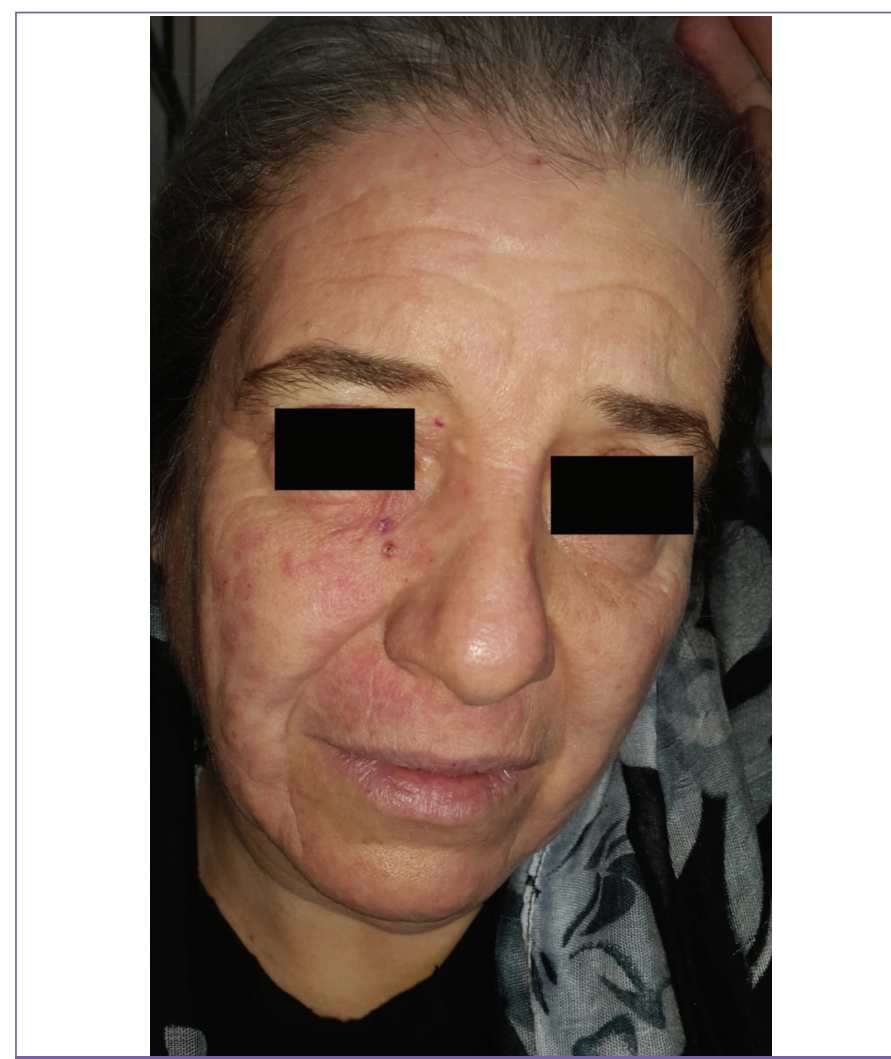

Figure 6. Rosacea like lesion of tinea in 52 years old female

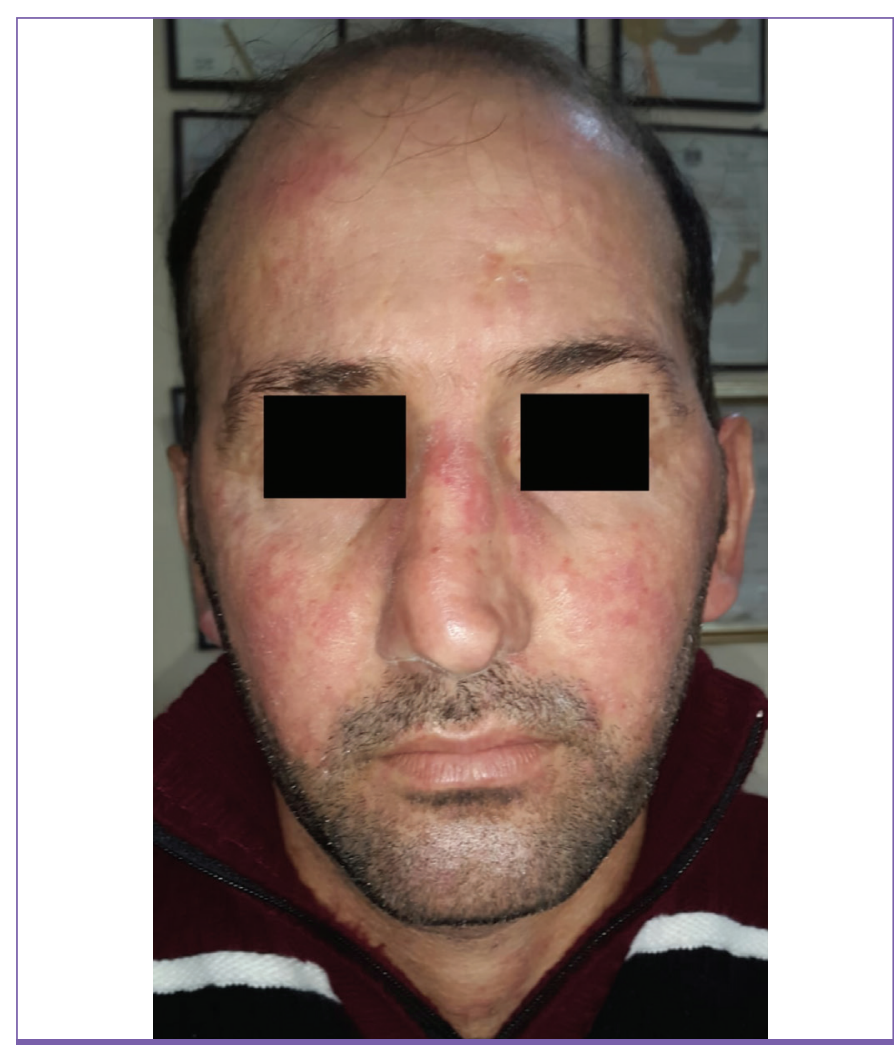

Figure 7. Tinea lesions on the malar area resembling lupus erythematosus in 29 years old male 


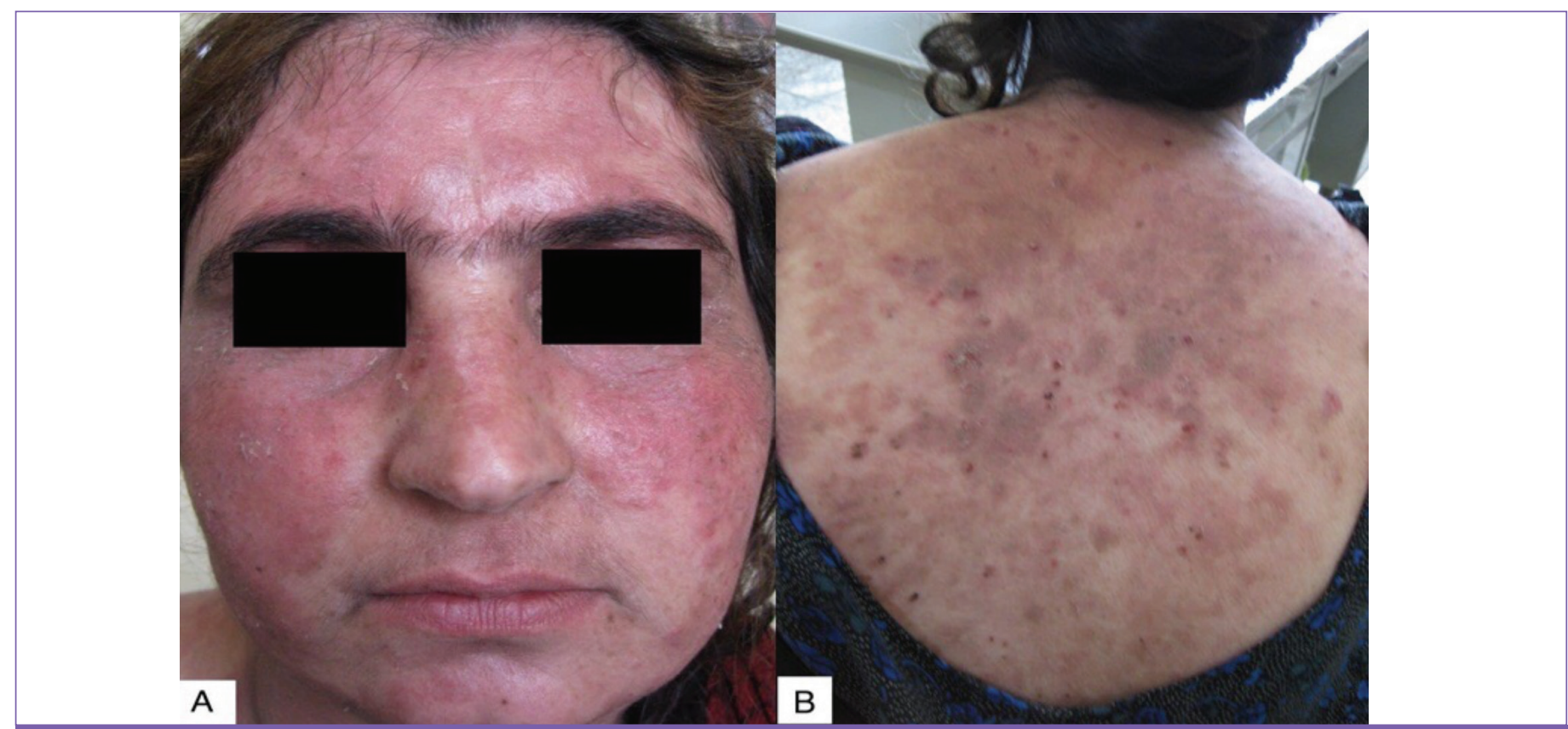

Figure 8. Pemphigus foliaceus like lesions of tinea on the face (A) and trunk (B) of 30 years old female

species were identified in specimens of 63 patients (out of 63 positive samples, 20 by PCR, 43 by culture): TM/TI, 40 (63\%, 26 by culture, 14 by PCR), M. canis, 7 (11\%, 6 by culture, 1 by PCR), E. floccosum, 6 (10\%, each 3 by culture and PCR, respectively), T. rubrum, 5 (8\%, 3 by culture, 2 by PCR), T. violaceum, 2 (3\%, 2 by culture). Each one strain of T. benhamiae (2\%), Nannizzia incurvata (2\%), and M. ferrugineum (2\%) was isolated.Depending on the results of sequencing, we were able to determine that among 26 cultural isolated TM strains, surprisingly, 18 were TM ITS type VIII India, 5 were TM ITS type V Iran, and 2 were belonging to anthropophilic TI ITS type II*. Seven (39\%) out of 18 Indian TM ITS type VIII were terbinafine resistant [24].

The atypical pictures of the following dermatophyte infections were as follow: tinea corporis was the main fungal infection observed in 175 (35\%) of cases followed by tinea faciei 124 (24.8\%), tinea manuum 75 (15\%), tinea capitis 59 (11.8\%), tinea pedis $32(6.4 \%)$, tinea cruris 28 (5.6\%), tinea unguium $4(0.8 \%)$ and tinea barbae 3 (0.6\%) (Table 1).

So the dermatophyte infections were mimicking the following skin diseases (Table 1): Psoriasiform lesions in 251 (50.2) patients, with careful history and examination, none had history of psoriasis currently or in the past, all of them tried various topical and in many, systemic treatments, with either no response at all or a notable exacerbation in size of lesions or in their numbers that terrifying the patients and provides an important clue to search for other diagnoses in favor of dermatophyte infections. On examination, the lesions were either resembling the thick classic plaque of psoriasis, or in some patients with minimally scaly lesions in moist flexural areas. Tinea corporis resembling resolved psoriatic lesions were also observed. The negative history of psoriasis with absence of response to various modalities of treatment was the clue to scrap these lesions and finally the accurate and treatable diagnosis of dermatophytosis was achieved.

Cutaneous dermatophyte infections presenting as dermatitis like lesions were the second most common atypical presentation affecting 107 (21.4\%) patients.

Similarly, while all patients received adequate treatment for misdiagnosed tinea infection as eczema, none of them were satisfied, and in majority, the lesions either enlarge or flare again upon discontinuation of topical steroid treatments.

In all patients who presented with photosensitivity like lesion and lupus like lesions of fungal infections, the antinuclear antibody and anti-double stranded DNA (anti-dsDNA) were negative and this was the guide for searching for an alternative diagnosis, with positive $\mathrm{KOH}$ examination that showed spores and hyphae, the cutaneous dermatophyte infection was the ultimate diagnosis.

\section{Discussion}

A worldwide increase in superficial dermatophyte infection of skin with varying degree of local inflammation had been reported in recent years [28].

While dermatophytes found universally, the prevalence rate may vary in different geographic areas depending on environmental conditions or lifestyle. Hot and humid weather, sun exposure, low socioeconomic status, overcrowding with sharing of clothes and 
Table 1. Atypical presentation, type of tinea, and sex of the patients in atypical variants of tinea infection

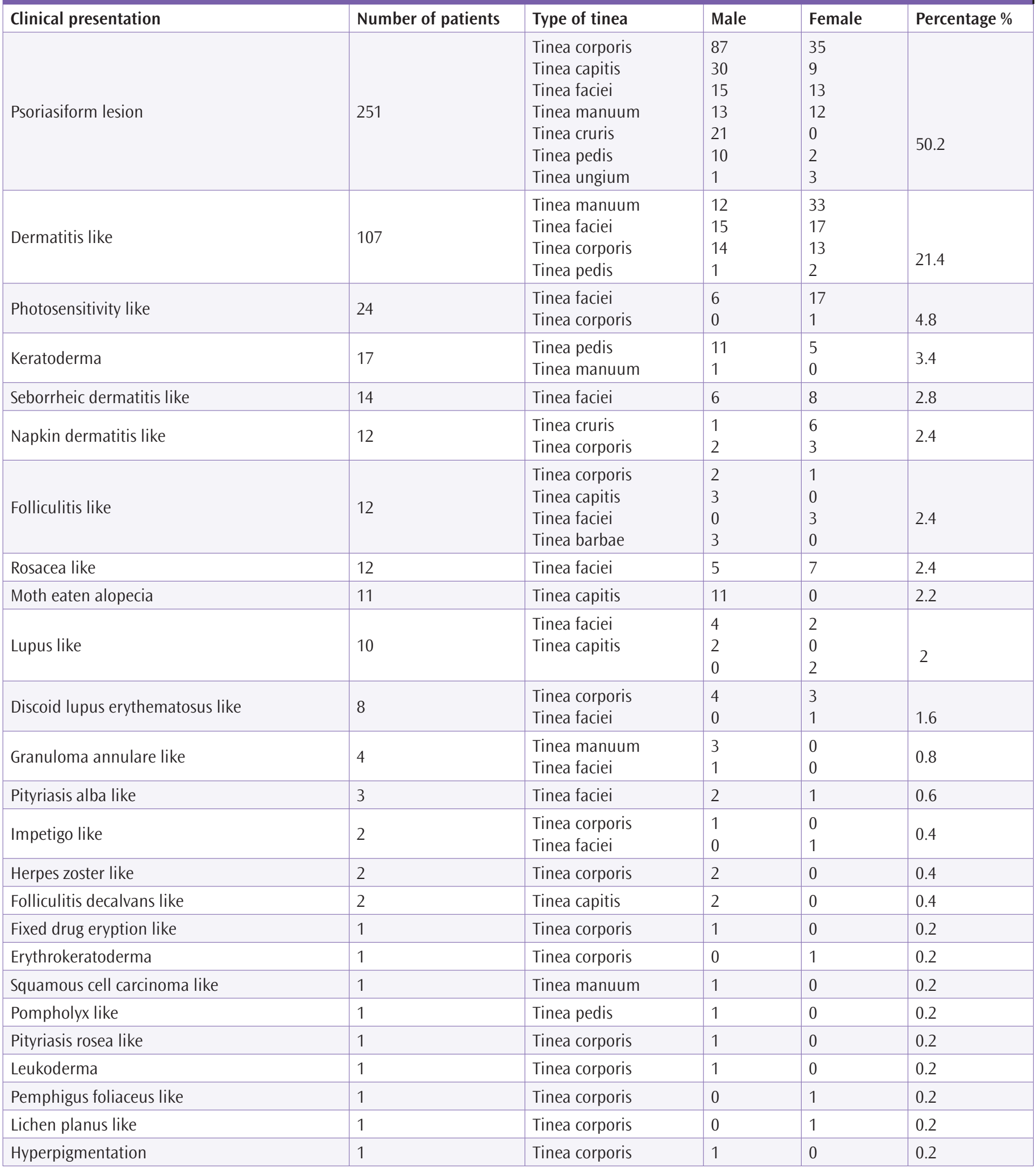

footwear, poor hygiene and sanitary conditions, and migration of population are important contributory factors for the increasing chronic and recurrent forms of dermatophytosis [18].

In the typical circumstances, the diagnosis of superficial dermatophyte infections is straight forward with no need for extensive or even simple laboratory investigations. Usually, the scaly erythematous patches in an annular arrangement, with peripheral enlargement and central clearing are the 
typical clinical presentation. However, when superficial cutaneous dermatophyte infections presented in a challenging clinical manifestations that is, totally or at least partially, nondistinguishable from other skin pathologies, it will result in delayed diagnosis as physicians do not include dermatophyte infections in their first diagnostic options. Many published literatures highlighted on atypical presentation of these infections [18,19,29,30,31,32,33,34,35].

Although the source of the infection is mainly animals, multiple infections between family members as a result of interpersonal contact within the family were observed in the present work. This observation could confirm that zoophilic species can be transmitted from one person to another causing dermatophytes infection.

In the present work, we introduced a large number of atypical superficial dermatophyte infections that were easily missed and wrongly diagnosed with other skin diseases and were considered as imitating skin diseases. These atypical mimicking presentations were also reported by other studies [18,20,29,30,31,32,33,34,35] but the present study was the most extensive evaluation.

Though rarely reported in Iraqi literature, the atypical clinical pictures of superficial fungal infection are now being routinely seen in different Iraqi cities. Tinea has now qualified for the group of great imitator similar to syphilis, cutaneous tuberculosis, sarcoidosis, and cutaneous leishmaniasis.

Psoriasis-like lesion in its classic plaque, flexural or early resolving lesions, was the most common clinical presentation (50.2\%) in our study which is inconsistent with another study in which eczema like lesion was the main clinical manifestation [20]. This observation may be due to variability in the clinical presentation of cutaneous fungal infection across the countries and even in the same country which can be attributed to host factors, climatic factors and dermatophyte species.

The present study recorded that TM/T. interdigitale (TI), (63\%) was the most common isolated causative species. While these results agreed with many earlier studies where TM was the most common isolate from the specimens [36,37], it disagreed with many previous reports in which M.canis was the main causative strain of dermatophytes infection $[20,38,39,40]$ while other studies $[24,41,42]$ showed that $\mathrm{T}$. rubrum was the main causative agents.

In this study, tinea corporis was the main fungal infection that observed in $35 \%$ of cases followed by tinea faciei $24.8 \%$, tinea manuum $15 \%$, tinea capitis $11.8 \%$, tinea pedis $6.4 \%$, tinea cruris $5.6 \%$, tinea unguium $0.8 \%$ and tinea barbae $0.6 \%$. These results were comparable to other Iraqi and international studies where tinea corporis and tinea capitis were the most prevalent clinical types $[10,12,43,44]$. The increased prevalence of tinea corporis and tinea capitis can be attributed to overcrowding, sharing of personal hygiene equipments including towels, combs and shavings tools [45] and rural drainage in many studied patients. In addition, some social behaviors in the Iraqi community, handshake can play a role in spreading infection as contact is an important portal for transmission of infection.

A statistically significant relation between dermatophytes infection and gender of the patients had been observed in the present study where the dermatophytes infection was higher in males with $59.6 \%$ cases, in comparison to females with $40.4 \%$ cases. Tinea corporis, tinea capities, tinea pedis, tinea cruris and tinea barbae had been recorded with higher frequency in males than females. While, tinea faciei, tinea manuum and tinea unguium displayed higher infections in females than males. The incidence of dermatophytosis in males was more than females in many earlier studies a finding in line with the present study $[12,37,46,47,48]$. On the other hand, the results inconsistent with other studies where the incidence of dermatophytosis was higher in females than males $[49,50,51]$. This difference between dermatophytes infection and gender of the patients may be due to physiological differences between male and female, life style and the differences in the social behavior [52].

Tinea capitis was the main fungal infection of preadolescent children. This is consistent with previous studies in Iraq [40,53] and other regions of the world $[10,54,55]$. Boys were affected more than girls this could be due to their outdoor playing and sport activities $[53,54,56,57]$. But tinea capitis was not uncommonly seen among adult patients as well documented by the present work.

Tinea cruris was more abundant in males (78.57\%) due to the presence of scrotum which provides warm and moist environment suitable for dermatophyte growth [58].

Tinea barbeae was only the disease of males (100\%) as it requires the hairy beard area of the patients.

On contrary, as the house duties are the responsibility of most women in our society, so it's not surprising to find higher frequency of tinea ungium in females (75\%) than males (25\%). Also, cosmetic manicuring practices undertaken by women can be considered as another explanation for this findings [59].

Surprisingly, in this study, TM constituted the most frequently recorded dermatophyte species in Iraq replacing T. rubrum, this observation is on line with the new emerged and mostly terbinafine resistant genotype VIII India. This can be explained by spread of TM type VIII-from India to neighboring Arab and Persian countries, e.g. Bahrain, and Iran, which facilitate their transmission to Iraq. The percentage of terbinafine resistance is high in the strains isolated in Baghdad, Iraq, so treatment failure of terbinafine are to be expected [24]. 
Early diagnosis of skin dermatophytosis is essential for all dermatologist particularly the lesion involving hairy areas as early and adequate treatment can prevent scarring outcome of this treatable conditions. While the atypical clinical presentation of cutaneous fungal infection described in the present study might be a new dilemma that can be added to many great imitator skin diseases, the customized and professional classification provided there, can help, at least in non-responding and recalcitrant lesions of common skin diseases, the clinician to think about of dermatophyte infection.

The ease of traveling, migration and overcrowding, displaced people which is a common problem in Iraq, the climatic conditions including high temperature and humidity and excessive washing with resultant modification of fungal growth and reproduction rate, and/or alteration in the skin barrier function, the abrupt increase in breeding pets, the incorrect diagnosis or treatment, and most importantly, the recently discovered TM type VIII in Iraqi patients with its chronic and recalcitrant nature with more ease of interfamily spread, these factors, all together, can provide robust explanation for the outbreak of dermatophyte infection in Iraq with atypical presentation.

The present work can draw an important observation that whenever the dermatophyte infection became chronic, the immunity against fungal pathogen will be very poor and the skin of these patients will behave like culture media allowing the fungal infection to spread quickly across the body skin.

\section{Study Limitations}

The present study showing large number of patients with well documented cases of atypical presentation of dermatophyte infections accordingly we think there is no limitation of the study.

\section{Conclusion}

The present study draws attention to the ability of tinea to imitate other cutaneous diseases, inviting the dermatologist not to simply trust the clinical point of view, especially when dealing with scaly erythematous dermatitis present for long duration and not respond to treatment especially topical steroids. Iraq is now running major outbreak of dermatophyte infections that have bizarre clinical pictures and mimicking many skin diseases commonly psoriasis, dermatitis, photosensivity and keratoderma. TM represented the most frequent dermatophyte that has been isolated.

\section{Ethics}

Ethics Committee Approval: The study followed the Declaration of Helsinki Principles and it was approved by the Ethics Committee of Fallujah Teaching Hospital (approval number: 1280, date: 16/9/2021).
Informed Consent: Oral consent was obtained from each patient after explanation of the nature of the study.

Peer-review: Externally and internally peer-reviewed.

\section{Authorship Contributions}

Surgical and Medical Practices: K.E.S., R.I.J., Concept: K.E.S., R.I.J., Design: K.E.S., R.I.J., Data Collection or Processing: K.E.S., R.I.J., Analysis or Interpretation: K.E.S., R.I.J., Literature Search: K.E.S., R.I.J., Writing: K.E.S., R.I.J.

Conflict of Interest: No conflict of interest was declared by the authors.

Financial Disclosure: The authors declared that this study received no financial support.

\section{References}

1. Lopes G, Pinto E, Salgueiro L. Natural products: An alternative to conventional therapy for dermatophytosis? Mycopathologia 2017;182:143-167.

2. Weitzman I, Summerbell RC. The dermatophytes. Clin Microbiol Re 1995;8:240-259

3. Havlickova B, Czaika VA, Friedrich M. Epidemiological trends in skin mycoses worldwide. Mycoses 2008 Sep;51 Suppl 4:2-15.

4. Sharquie KE, Al-Zubaidi KA. Microsporum canis in Iraq. Saudi Med J 1985;6:248-250.

5. Clayton YM, Midgley G. Tinea capitis bei Schulkindern in London [Tinea capitis in school children in London]. Hautarzt 1977;28:32-34.

6. Nowicki R. Dermatophytoses in the Gdańsk area, Poland: a 12-year survey. Mycoses 1996;39:399-402.

7. Buchvald J, Simaljaková M. The occurrence of dermatophytes in Slovakia. Mycoses 1995;38:159-161.

8. Korstanje MJ, Staats CC. Fungal infections in the Netherlands. Prevailing fungi and pattern of infection. Dermatology 1995;190:39-42.

9. Nweze El. Dermatophytosis in Western Africa: a review. Pak J Biol Sci 2010;13:649-656.

10. Ellabib MS, Khalifa Z, Kavanagh K. Dermatophytes and other fungi associated with skin mycoses in Tripoli, Libya. Mycoses 2002;45:101-104.

11. Sharquie KE, Mowlud AK. A survey of superficial fungal infections of skin Iraqi Med J 1993;40:41-43.

12. Najem MH, Al-Salhi MH, Hamim SS. Study of Dermatophytosis prevalence in Al-Nassiriyah city- Iraq. World J Pharm Sci 2016;4:166-172.

13. al-Fouzan AS, Nanda A, Kubec K. Dermatophytosis of children in Kuwait: a prospective survey. Int J Dermatol 1993;32:798-801.

14. Ali-Shtayeh MS, Arda HM. A study of tinea capitis in Jordan (West Bank). J Trop Med Hyg 1986;89:137-141.

15. Venugopal PV, Venugopal TV. Tinea capitis in Saudi Arabia. Int J Dermatol 1993;32:39-40

16. Maraki S, Tselentis Y. Survey on the epidemiology of Microsporum canis infections in Crete, Greece over a 5-year period. Int J Dermatol 2000;39:21-24.

17. Hay RJ, Robles W, Midgley G, Moore MK; European Confederation of Medical Mycology Working Party on Tinea Capitis. Tinea capitis in Europe: new perspective on an old problem. J Eur Acad Dermatol Venereol 2001;15:229233. 
18. Dogra S, Narang T. Emerging atypical and unusual presentations of dermatophytosis in India. Clin Dermatol Rev 2017;1:12-18.

19. Sahoo AK, Mahajan R. Management of tinea corporis, tinea cruris, and tinea pedis: A comprehensive review. Indian Dermatol Online J 2016;7:77-86.

20. Atzori L, Pau M, Aste N, Aste N. Dermatophyte infections mimicking other skin diseases: a 154-person case survey of tinea atypica in the district of Cagliari (Italy). Int J Dermatol 2012;51:410-415.

21. Verma S, Madhu R. The Great Indian Epidemic of Superficial Dermatophytosis: An Appraisal. Indian J Dermatol 2017;62:227-236.

22. Verma S, Vasani R, Reszke R, Matusiak Ł, Szepietowski JC. Prevalence and clinical characteristics of itch in epidemic-like scenario of dermatophytoses in India: a cross-sectional study. J Eur Acad Dermatol Venereol 2020;34:180183.

23. Nenoff P, Verma SB, Ebert A, Süß A, Fischer E, Auerswald E, Dessoi S, Hofmann W, Schmidt S, Neubert K, Renner R, Sohl S, Hradetzky U, Krusche U, Wenzel HC, Staginnus A, Schaller J, Müller V, Tauer C, Gebhardt M, Schubert K, Almustafa Z, Stadler R, Fuchs A, Sitaru C, Retzlaff C, Overbeck C, Neumann T, Kerschnitzki A, Krause S, Schaller M, Walker B, Walther T, Köhler L, Albrecht M, Willing U, Monod M, Salamin K, Burmester A, Koch D, Krüger C, Uhrlaß S. Spread of Terbinafine-Resistant Trichophyton mentagrophytes Type VIII (India) in Germany-"The Tip of the Iceberg?". J Fungi (Basel) 2020;6:207.

24. Uhrlass S, Alhilaifawi AFH, Sharquie KE, Alhamami HR, Al-Qahar GHA, Tabra MKH, Koch D, Muetze H, Kruege C, Burmester A, Wiegand C, Hipler UC. Dermatophyte survey from Baghdad, Iraq - emergence of terbinafine resistant Trichophyton mentagrophytes Type VIII India. Submitted (MAY2020) to the EMBL/GenBank/DDBJ databases.

25. Järv H, Uhrlaß S, Simkin T, Nenoff P, Alvarado RE; Chryssanthou E, Monod M. Terbinafine resistant Trichophyton mentagrophytes genotype VIII, Indian type, isolated in Finland. J Fungi 2019;5:117.

26. Uhrlass S, Sithach M, Koch D, Wittig F, Muetze H, Krueger C, Nenoff P. Trichophyton mentagrophytes-A new genotype in Cambodia. J Fungi 2019;5:460.

27. Taghipour S, Shamsizadeh F, Pchelin IM, Rezaei-Matehhkolaei A, Zarei Mahmoudabadi A, Valadan R, Ansari S, Katiraee F, Pakshir K, Zomorodian K, Abastabar M. Emergence of Terbinafine Resistant Trichophyton mentagrophytes in Iran, Harboring Mutations in the Squalene Epoxidase (SQLE) Gene. Infect Drug Resist 2020;13:845-850.

28. Yadav A, Urhekar AD, Mane V, Danu MS, Goel N, Ajit KG. Optimization andisolation of dermatophytes from clinical samples and in vitro antifungal susceptibility testing by disc diffusion method. Research \& Reviews: Journal of Microbiology and Biotechnology 2013;2:19-34.

29. Serarslan G. Pustular psoriasis-like tinea incognito due to Trichophyton rubrum. Mycoses 2007;50:523-524.

30. Romano C, Maritati E, Gianni C. Tinea incognito in Italy: a 15-year survey. Mycoses 2006;49:383-387.

31. Meymandi S, Wiseman MC, Crawford RI. Tinea faciei mimicking cutaneous lupus erythematosus: a histopathologic case report. J Am Acad Dermatol 2003;48:57-8.

32. Ghislanzoni M. Tinea incognito due to Trichophyton rubrum responsive to topical therapy with isoconazole plus corticosteroid cream. Mycoses 2008;51 Suppl 4:39-41.

33. Nenoff $P$, Mügge $C$, Herrmann J, Keller U. Tinea faciei incognito due to Trichophyton rubrum as a result of autoinoculation from onychomycosis. Mycoses 2007;50 Suppl 2:20-25.

34. Atzori L, Pau M, Aste M. Erythema multiforme ID reaction in atypical dermatophytosis: a case report. J Eur Acad Dermatol Venereol 2003;17:699701.
35. Aste N, Pau M, Aste N, Atzori L. Tinea corporis mimicking herpes zoster. Mycoses 2011;54:463-465.

36. Sowmya N, Appalaraju B, Surendran P, Srinivas CR. Isolation, Identification and comparatative analysis of SDA and DTM for dermatophytes from clinical samples in a tertiary care hospital. IOSR Journal of Dental and Medical Sciences 2014;13:68-73.

37. Rashidian S, Falahati M, Kordbacheh P, Mahmoudi M, Safara M, Sadeghi Tafti H, Mahmoudi S, Zaini F. A study on etiologic agents and clinical manifestations of dermatophytosis in Yazd, Iran. Curr Med Mycol 2015;1:2025.

38. Ndako JA, Osemwegie OO, Spencer THI, Olopade BK, Yunusa GA, Banda J. Prevalence of Dermatophytes and other associated fungi among school children. Global Advanced Research Journal of Medicine and Medical Sciences 2012;1:49-56.

39. Veasey JV, Miguel BAF, Mayor SAS, Zaitz C, Muramatu LH, Serrano JA. Epidemiological profile of tinea capitis in São Paulo City. An Bras Dermatol 2017;92:283-284.

40. Sharquie KE, Al Ani SM, Ali TM. Microsporum canis epidemic in Iraq children. Journal of the Faculty of Medicine-Baghdad 1992;34:95-99.

41. Mohammed SJ, Noaimi AA, Sharquie KE. A Survey Of Dermatophytes Isolated From Iraqi Patients In Baghdad City. AL-Qadisiya Medical Journal 2015;11:1015.

42. Toukabri N, Dhieb C, El Euch D, Rouissi M, Mokni M, Sadfi-Zouaoui N. Prevalence, Etiology, and Risk Factors of Tinea Pedis and Tinea Unguium in Tunisia. Can J Infect Dis Med Microbiol 2017;2017:6835725.

43. Mahmoud AL. A study of dermatophytoses in Sana'a, Yemen Republic. Mycoses 2002;45:105-108.

44. Lange M, Nowicki R, Bara冈ska-Rybak W, Bykowska B. Dermatophytosis in children and adolescents in Gdansk, Poland. Mycoses 2004;47:326-329.

45. Brook GF, Butel JS, Morse SA, Jawetz and Adelbergs medical microbiology. 2nd. Middle East. Beirut; Lebanon: 2001.

46. Morar N, Dlova NC, Gupta AK, Aboobaker J. Tinea capitis in Kwa-Zulu Natal, South Africa. Pediatr Dermatol 2004;21:444-447.

47. Rahman MH. Hadiuzzaman MD, Bhuiyan MKJ, Islam N, Ansari NP, Mumu SA, Chowdhury IJ. Prevalence of superficial fungal infections in the rural areas of Bangladesh. Iran J Dermatol 2011;14:86-91.

48. Sahin I, Oksuz S, Kaya D, Sencan I, Cetinkaya R. Dermatophytes in the rural area of Duzce, Turkey. Mycoses 2004;47:470-474.

49. Brajac I, Stojni囚-Sosa L, Prpi囚 L, Loncarek K, Gruber F. The epidemiology of Microsporum canis infections in Rijeka area, Croatia. Mycoses 2004;47:222226.

50. Brilhante RS, Cordeiro RA, Rocha MF, Monteiro AJ, Meireles TE, Sidrim JJ. Tinea capitis in a dermatology center in the city of Fortaleza, Brazil: the role of Trichophyton tonsurans. Int J Dermatol 2004;43:575-579.

51. Vella Zahra L, Gatt P, Boffa MJ, Borg E, Mifsud E, Scerri L, Vella Briffa D, Pace JL. Characteristics of superficial mycoses in Malta. Int J Dermatol 2003;42:265271.

52. Hay RJ, Adrians BM. Bacterial infection. In: Champion RH, Burton JL, Burns DA, Breatandem JM, editors. Textbook of Dermatology, 6th ed, London: Blackwell Sciences; 1998. p. 1113-1116.

53. Abed Ali FAH, Al-Janabi JKA, Mohammed K, Alhattab MK. Prevalence of dermatophyte fungal infection in Hillah, Iraq. International Journal of Chem Tech Research 2017;10:827-837.

54. Naseri A, Fata A, Najafzadeh MJ, Shokri H. Surveillance of dermatophytosis in northeast of Iran (Mashhad) and review of published studies. Mycopathologia 2013;176:247-253. 
55. Maulingkar SV, Pinto MJ, Rodrigues S. A clinico-mycological study of dermatophytoses in Goa, India. Mycopathologia 2014;178:297-301.

56. Moneib HA, El-Shiemy SMH, Saudi WM, El-Fangary MM, Nabil T, Mohy SM. Hair loss among a group of Egyptian children: a clinical and dermoscopic study. J Egypt Women Dermatol Soc 2017;14:9-24.

57. Nnoruka EN, Obiagboso I, Maduechesi C. Hair loss in children in South-East Nigeria: common and uncommon cases. Int J Dermatol 2007;46(Suppl 1):18-22.
58. Abdel-Rahman SM, Farrand N, Schuenemann E, Stering TK, Preuett B, Magie R, Campbell A. The prevalence of infections with Trichophyton tonsurans in schoolchildren: the CAPITIS study. Pediatrics 2010;125:966973.

59. Olde Hartman TC, van Rijswijk E. Fungal nail infection. BMJ 2008;337:a429. 